\title{
Aperçu
}

\section{Hospitalisations pour blessure au Canada en 2018-2019}

\author{
Xiaoquan Yao, M. Sc.; Robin Skinner, MSP; Steven McFaull, M. Sc.; Wendy Thompson, M. Sc.
}

Diffuser cet article sur Twitter

\begin{abstract}
Résumé
Les statistiques nationales sur les hospitalisations pour blessure sont essentielles pour comprendre le fardeau et le profil des blessures. Dans cette étude, on a utilisé la Base de données sur les congés des patients afin d'analyser les hospitalisations pour blessure au Canada (à l'exception du Québec) pour l'exercice 2018-2019. Il en ressort que les blessures non intentionnelles occupent le huitième rang des principales causes d'hospitalisation pour l'ensemble des maladies et affections. Dans le cas des hospitalisations pour blessure non intentionnelle, les principales causes sont, dans l'ordre, les chutes, la suffocation, les accidents de la circulation avec un véhicule à moteur, les empoisonnements, l'exposition à des forces mécaniques et l'exposition à la fumée, au feu et aux objets brûlants. Toutefois, ce classement est variable selon le groupe d'âge.
\end{abstract}

Mots-clés : hospitalisations pour blessure, principales causes, blessures non intentionnelles, blessures auto-infligées, agressions, chutes

\section{Introduction}

Une blessure est un transfert d'énergie à l'être humain à une vitesse et en une quantité au-delà ou en deçà de la gamme de tolérance du tissu humain ${ }^{1,2}$. Il est possible de regrouper les blessures en fonction de la cause externe, qui correspond approximativement au type d'énergie : chutes (mécanique), accidents de la circulation avec un véhicule à moteur (mécanique), exposition à des forces mécaniques (mécanique), empoisonnement (chimique), suffocation (asphyxie, manque d'énergie), exposition à la fumée, au feu et aux objets brûlants (thermique ou chimique) et autres (divers types d'énergie).

On peut aussi classer les blessures en blessures intentionnelles et blessures non intentionnelles. Les blessures non intentionnelles ne sont pas infligées volontairement ni dans l'intention de causer des lésions. Citons en exemple les trébuchements suivis d'une chute ou encore un accident de la circulation avec un véhicule à moteur ${ }^{3,4}$. Les blessures intentionnelles découlent d'un acte délibéré, envers soi (auto-infligées) ou infligé par autrui (agression). Lorsque l'intention n'est pas claire, la blessure est considérée comme ayant une intention indéterminée. L'intention et la cause externe aident à comprendre le mécanisme des blessures, établissant les assises de la prévention des blessures.

Les blessures constituent un problème de santé publique : elles ont causé 4,9 millions de décès à l'échelle mondiale en $2016^{5}$ et un nombre beaucoup plus élevé d'hospitalisations, de visites à l'urgence et de consultations médicales ${ }^{6}$. Au Canada, en 2018, 17843 personnes sont mortes à la suite de blessures ${ }^{7}$ et, durant l'exercice 2017-2018, plus de 269000 hospitalisations ont eu lieu pour blessure ${ }^{8}$. Le fardeau économique associé aux blessures en 2010 se chiffrait à environ 27 milliards de dollars canadiens ${ }^{9}$.

Les efforts de prévention des blessures reposent sur une compréhension globale et actualisée du fardeau et du profil des blessures au Canada. Ainsi, Parachute, un organisme national de prévention des blessures, établit ses priorités stratégiques ${ }^{10}$ et rédige ses rapports sur le fardeau économique et le coût des blessures en

\section{Points saillants}

- Les statistiques nationales sur les hospitalisations pour blessure permettent de comprendre le fardeau et le profil des blessures, en particulier les incidents non mortels, afin d'orienter les stratégies de prévention au Canada.

- Pour l'exercice 2018-2019, les blessures non intentionnelles occupent le huitième rang des principales causes d'hospitalisation pour l'ensemble des maladies et affections. Ce type de blessure se classe au neuvième rang ou à un rang supérieur en tant que principale cause d'hospitalisation pour tous les groupes d'âge sauf les enfants de moins d'un an.

- Les chutes sont la principale cause d'hospitalisation pour blessure non intentionnelle, tous groupes d'âge confondus.

s'appuyant sur des données de ce type ${ }^{9}$. Notre article de 2019 indiquait que les blessures non intentionnelles étaient soit la première soit la deuxième cause de décès chez les 1 à 44 ans et que le suicide était la deuxième cause de décès chez les 15 à 34 ans $^{11}$.

Les statistiques sur les hospitalisations permettent de comprendre le fardeau des blessures, en particulier des incidents non mortels. Les statistiques sur les visites à l'urgence et les hospitalisations à la suite d'un traumatisme ou d'une blessure pour l'exercice 2017-2018, publiées par l'Institut canadien d'information sur la santé (ICIS), fournissent les taux d'hospitalisation pour blessure par province ou territoire au Canada et le nombre d'hospitalisations 
pour divers types de blessure selon la cause et l'intention ${ }^{8}$. Dans une étude menée en 2013, l’Agence de la santé publique du Canada (ASPC) a adopté une autre approche de présentation des statistiques nationales sur les hospitalisations pour blessure : elle a classé les hospitalisations pour divers types de blessures (non intentionnelles, auto-infligées et agression) par rapport à d'autres maladies et affections ${ }^{12}$. Cette étude classait également les hospitalisations en fonction des principales causes externes de blessure ${ }^{13}$. Cette présentation des données fournit un portrait clair du fardeau relatif des grands groupes de blessures et peut servir de complément aux statistiques de l'ICIS.

Les objectifs de notre étude sont d'actualiser les tableaux antérieurs à l'aide des données disponibles les plus récentes pour le Canada (2018-2019, à l'exception du Québec) et d'illustrer le fardeau et le profil des hospitalisations pour blessure, afin d'éclairer les décisions en matière de prévention des blessures.

\section{Méthodologie}

Les données de cette étude sont tirées de la Base de données sur les congés des patients (BDCP) 2018-2019 de l'ICIS, qui n’inclut pas les hôpitaux du Québec. Dans le cadre de cette étude, nous n’avons retenu que les congés des établissements de soins de courte durée, pour lesquels nous utilisons le terme " hospitalisation ». Au total, nous avons sélectionné 2587663 enregistrements de patients hospitalisés en soins de courte durée ayant reçu leur congé entre le $1^{\text {er }}$ avril 2018 et le 31 mars 2019, après exclusion des mortinaissances, des cadavres et des enregistrements en double. Le nombre d'enregistrements correspond au nombre de congés et non au nombre de patients.

Les diagnostics de la BDCP 2018-2019 ont été codifiés selon la CIM-10-CA (Classification statistique internationale des maladies et des problèmes de santé connexes, $10^{\mathrm{e}}$ version, Canada) ${ }^{14}$. Nous avons utilisé la variable du diagnostic principal pour établir la cause de l'hospitalisation et comparer le fardeau des blessures non intentionnelles, des blessures auto-infligées et des agressions à celui d'autres maladies ou affections. Si le code du diagnostic principal commence par « S » ou " $\mathrm{T}$ », l'enregistrement est un enregistrement de blessure, qui est ensuite classé, en fonction du code de cause externe du diagnostic, comme blessure non intentionnelle, blessure auto-infligée, agression, blessure avec intention indéterminée ou autre. Si l'enregistrement compte plusieurs causes externes, la classification est effectuée selon l'ordre de priorité suivant : agression, blessures auto-infligées, blessures non intentionnelles, blessures avec intention indéterminée et autres.

Nous avons réalisé des analyses complémentaires sur les hospitalisations pour blessure. Afin d'inclure toutes les hospitalisations pour blessure, nous avons fait des recherches sur tous les codes de cause externe, indépendamment de la présence de " $\mathrm{S}$ » ou de « $\mathrm{T}$ » dans le code de diagnostic principal. Nous avons classé les blessures en fonction de l'intention, puis, pour les blessures non intentionnelles, de la cause externe (chutes, suffocation, accidents de la circulation avec un véhicule à moteur, empoisonnement, exposition à des forces mécaniques, exposition à la fumée, au feu et aux objets brûlants). Nous avons omis les complications liées aux soins médicaux et chirurgicaux; celles-ci n'étaient pas visées par la portée de notre étude, en raison de leur nature et de leurs modalités de prévention distinctes $^{3}$. Lorsqu'un enregistrement était associé à plusieurs groupes de blessures, il a été comptabilisé plusieurs fois.

Nous avons utilisé SAS Enterprise Guide version $71^{15}$ pour compiler les dénombrements regroupés et stratifiés (selon le sexe et l'âge). Nous avons utilisé les estimations de la population (à l'exclusion du Québec) au 1er octobre 2018 de Statistique Canada ${ }^{16}$ pour le calcul des taux bruts.

\section{Résultats}

\section{Principales causes d'hospitalisation}

Les principales causes d'hospitalisation sont présentées dans le tableau 1. Par rapport à toutes les autres maladies et affections (dont les hospitalisations pour examens, soins spécifiques, risques potentiels et motifs liés à la reproduction et divers symptômes, signes et constatations impossibles à classer), les blessures non intentionnelles occupent le huitième rang au classement général, le septième rang chez les hommes et le neuvième rang chez les femmes. Pour tous les groupes d'âge, sauf pour les enfants de moins d'un an, ces blessures se classent au neuvième rang ou à un rang supérieur. Elles comptent parmi les cinq premières causes d'hospitalisation chez les 1 à 34 ans et chez les 80 ans et plus. Les blessures auto-infligées arrivent au neuvième rang des principales causes d'hospitalisation chez les 15 à 19 ans et au dixième rang chez les 20 à 24 ans.

\section{Principales causes d'hospitalisations pour blessure}

Le tableau 2 illustre que, dans l'ensemble, les taux d'hospitalisation suivent un ordre similaire chez les hommes et les femmes en ce qui concerne les blessures non intentionnelles, mais des divergences sont visibles dans le cas des blessures intentionnelles. Le taux d'hospitalisation pour chute accidentelle ou blessure autoinfligée est plus élevé chez les femmes. En revanche, le taux d'hospitalisation est plus élevé chez les hommes dans les catégories suffocation accidentelle, accident de la circulation avec un véhicule à moteur, empoisonnement, exposition à des forces mécaniques, exposition à la fumée, au feu et aux objets brûlants et agression.

Pour tous les âges confondus, le classement des blessures non intentionnelles par cause externe (du taux le plus élevé au taux le plus faible) est : chutes, suffocation, accident de la circulation avec un véhicule à moteur, empoisonnement, exposition à des forces mécaniques et exposition à la fumée, au feu et aux objets brûlants. Globalement, le taux d'hospitalisation pour chute augmente considérablement à partir de 65 ans, passant à 4 fois puis à 16 fois le taux observé chez les 45 à 64 ans.

Outre les chutes, qui sont la principale cause d'hospitalisation pour blessure non intentionnelle tous groupes d'âge confondus, nous constatons des variations dans le classement des causes externes selon le groupe d'âge. La suffocation est la deuxième cause en importance chez les enfants de moins de 10 ans et chez les adultes de 45 ans et plus. Les accidents de la circulation avec un véhicule à moteur occupent le deuxième rang des principales causes chez les 15 à 44 ans. Chez les enfants de 10 à 14 ans, la deuxième position revient à l'exposition à des forces mécaniques.

Il convient de souligner que, chez les 15 à 24 ans, le taux d'hospitalisation pour blessure auto-infligée dépasse celui pour chute non intentionnelle. Le taux d'hospitalisation 


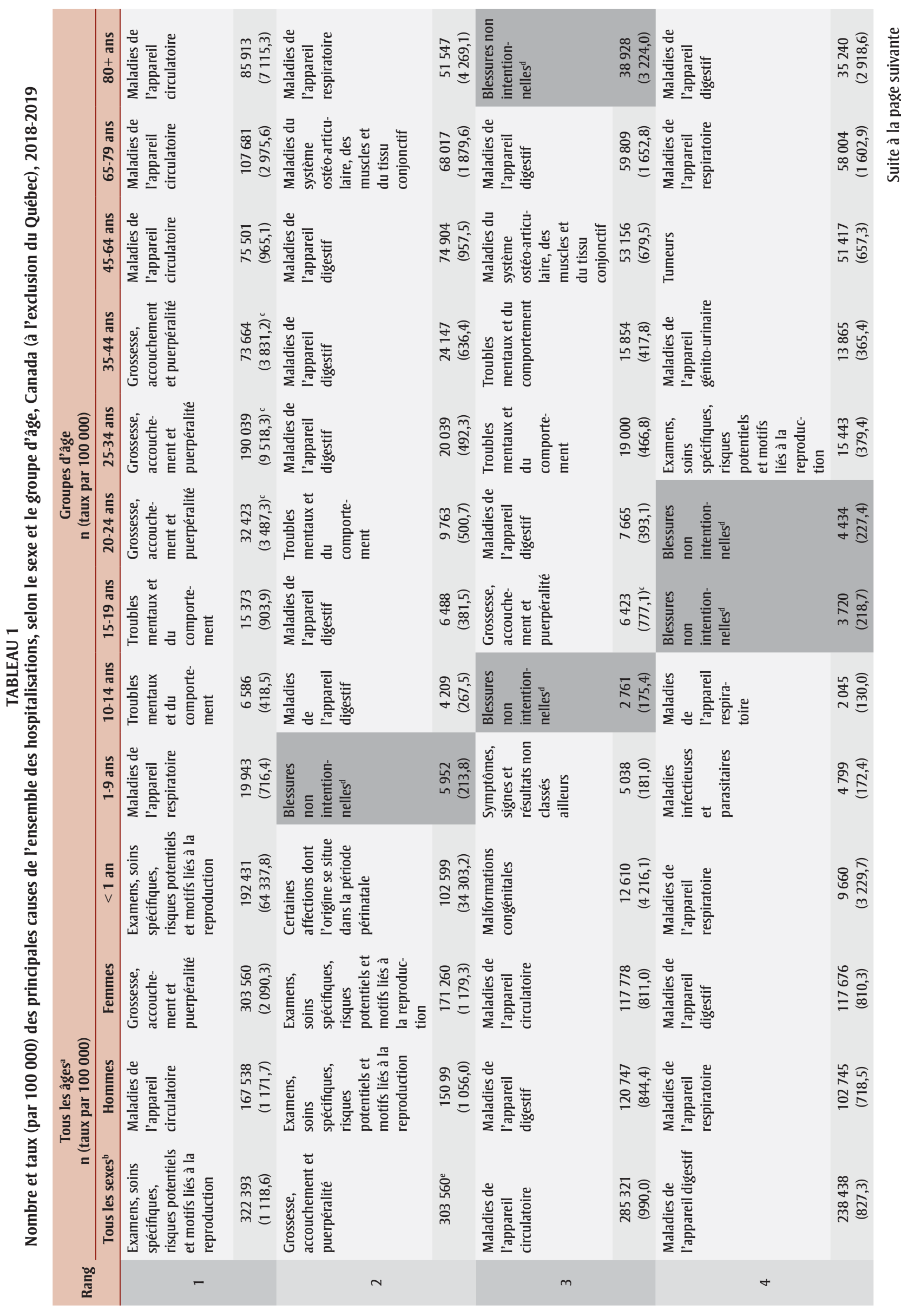




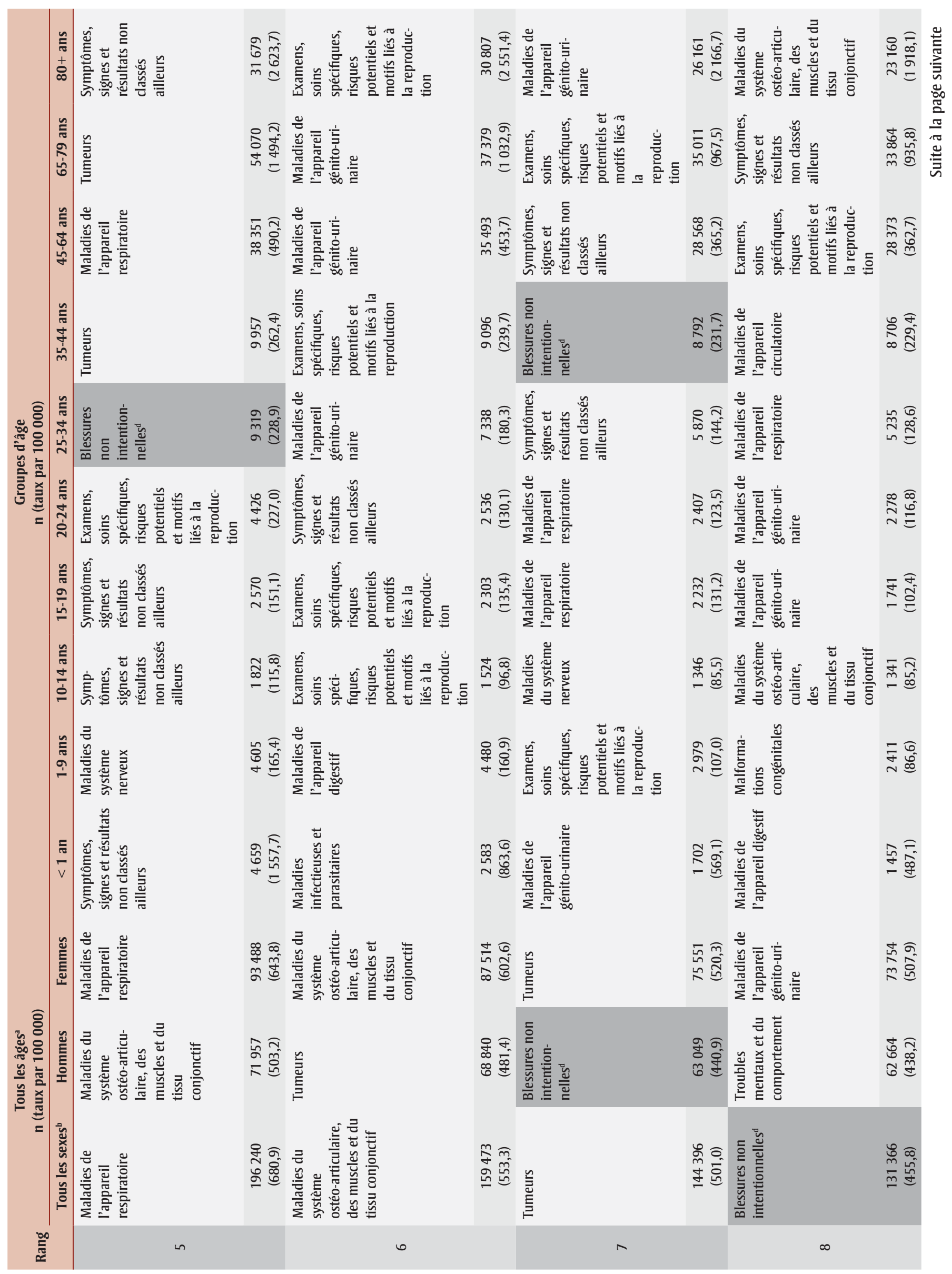




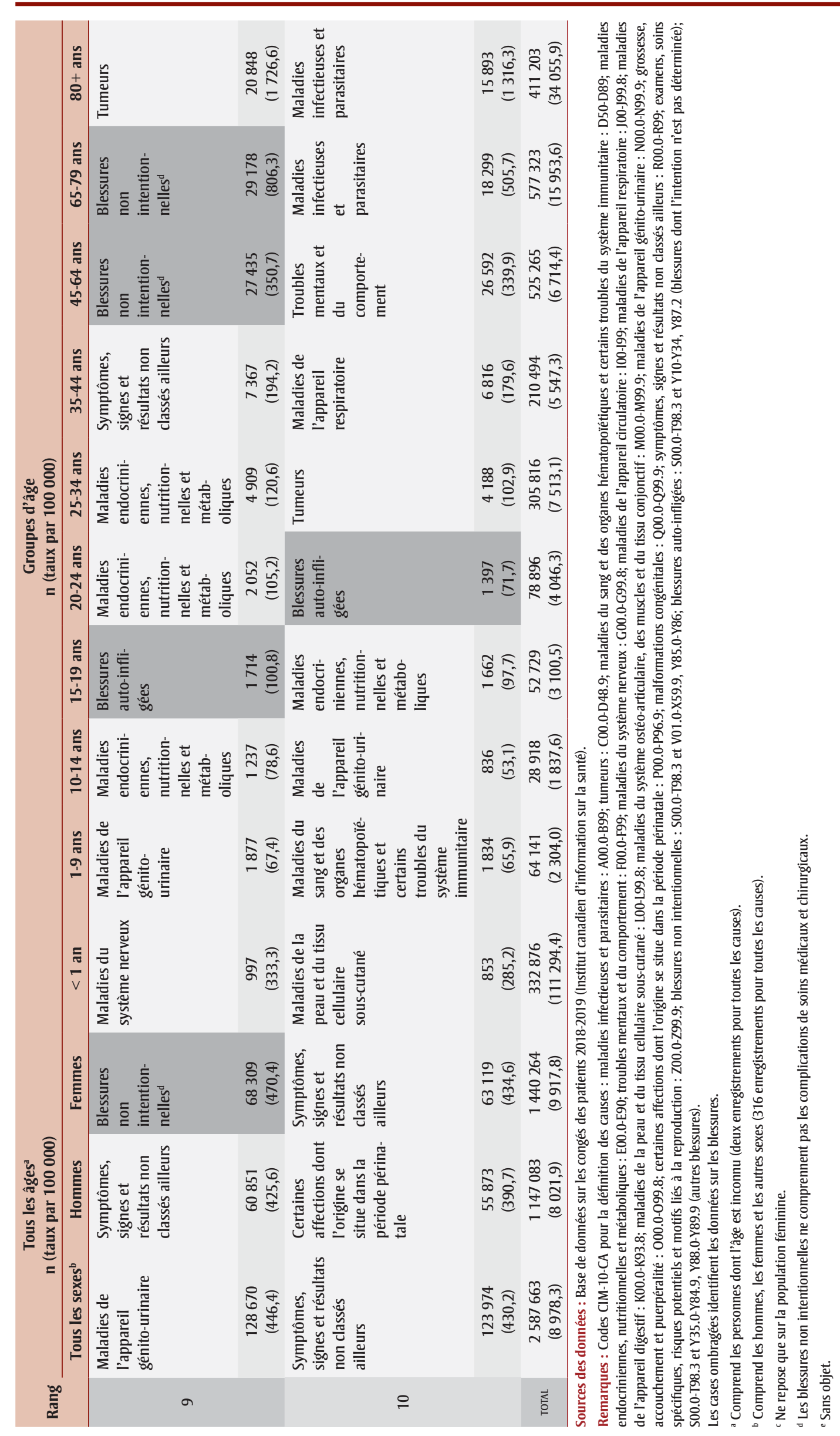




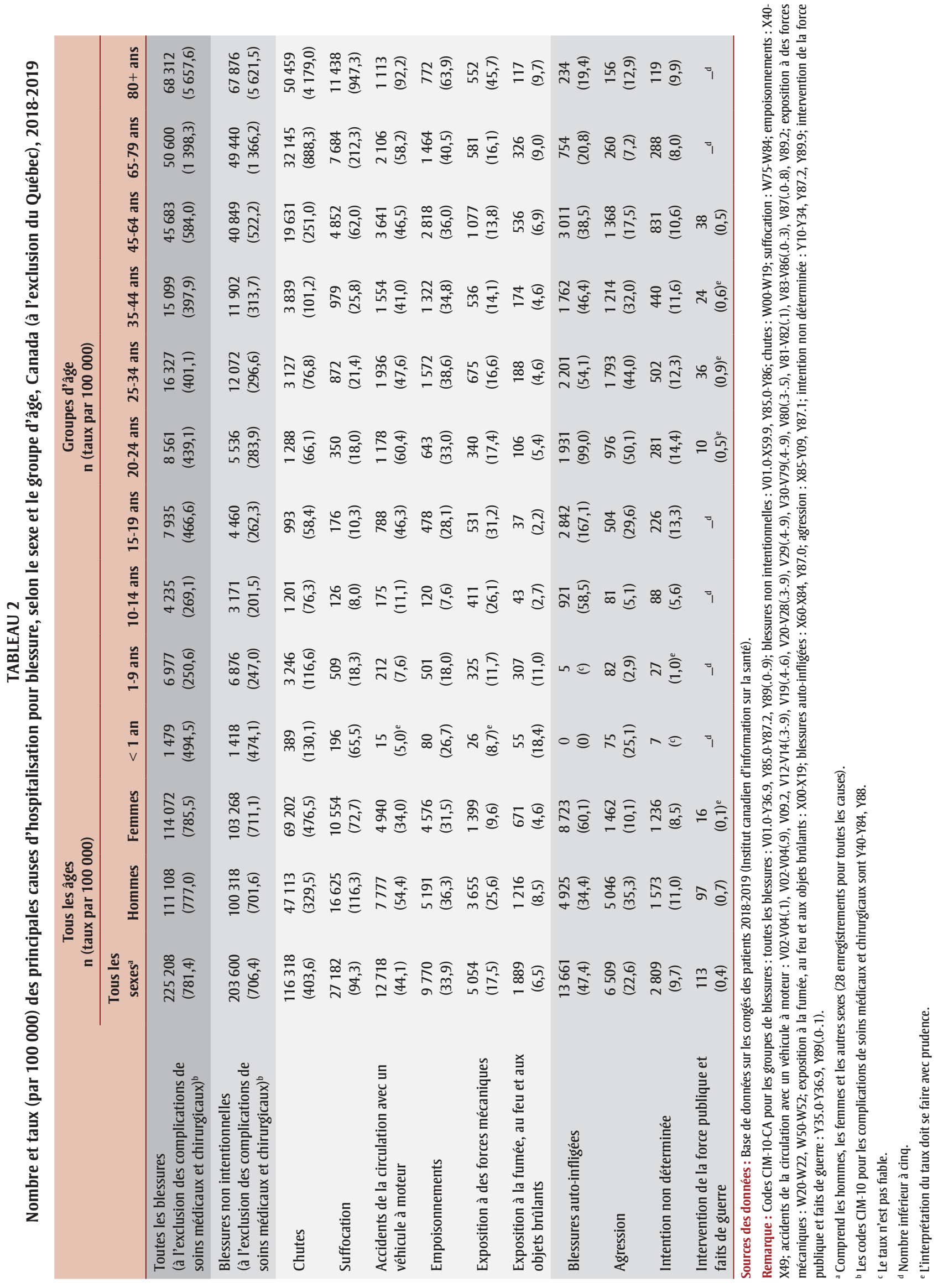


pour agression le plus élevé est observé quant à lui chez les adultes de 20 à 24 ans.

\section{Analyse}

Cette étude fournit les statistiques nationales les plus récentes sur les hospitalisations pour blessure, ce qui permet d'aider à comprendre le fardeau et le profil des blessures au Canada.

Les résultats révèlent que, globalement, les blessures non intentionnelles sont la huitième cause d'hospitalisation durant l'exercice 2018-2019. Les chutes occupent le premier rang des causes d'hospitalisation pour blessure non intentionnelle, tous groupes d'âge confondus, et le taux augmente particulièrement chez les aînés (65 ans et plus), passant à 4 fois puis à 16 fois le taux des personnes d'âge moyen. Comme les chutes chez les aînés imposent un énorme fardeau au système de santé canadien ${ }^{9}$ et que le vieillissement des baby-boomers accroît la proportion de la population âgée de 65 ans et plus, il est très important de suivre de près l'évolution des chutes au sein de cette tranche de la population pour dégager les tendances et élaborer des programmes de prévention efficaces $^{17}$. Les variations dans le classement des autres groupes de blessures non intentionnelles selon les groupes d'âge montrent d'ailleurs l'importance d'adapter les efforts de prévention en fonction des groupes d'âge. Ces données vont pouvoir servir de base à la planification stratégique et aux messages publics en matière de prévention des blessures ${ }^{10,18}$.

Les blessures auto-infligées et les agressions chez les jeunes sont également un important problème de santé publique. Les blessures auto-infligées servent à établir les tentatives de suicide, et le taux élevé d'hospitalisation pour blessure autoinfligée chez les 15 à 24 ans doit faire l'objet de recherches plus approfondies. Le groupe affichant le taux d'hospitalisation pour agression le plus élevé est celui des 20 à 24 ans. Ces résultats soulignent l'importance d'accentuer les mesures de prévention auprès des adolescents et des jeunes adultes ${ }^{19,20}$.

\section{Limites}

La population du Québec représente $22,6 \%$ de la population canadienne ${ }^{16}$. Notre source d'information, la BDCP de l'ICIS, n'inclut pas les données sur les hospitalisations dans cette province. Le ministère de la Santé et des Services sociaux du Québec transmet les statistiques sur les hospitalisations selon le sexe (sans répartition par groupe d'âge) par l'entremise de MED-ÉCHO ${ }^{21}$, mais nos analyses nécessitent l'usage de microdonnées.

Par ailleurs, dans le but de comparer le fardeau des blessures à celui des autres maladies et affections, nous avons utilisé à la fois les diagnostics principaux (codes $\mathrm{S}$ et $\mathrm{T}$ ) et les codes de cause externe pour repérer les cas de blessures. Cette méthodologie classe les enregistrements dont la cause externe est la cause sous-jacente du diagnostic principal sans blessure dans la catégorie des cas sans blessure. Par conséquent, elle sous-estime les cas de blessures. Ainsi, les nombres de blessures non intentionnelles, auto-infligées et autres ne peuvent être égaux dans les tableaux 1 et 2 .

La méthode d'analyse des données peut également avoir une influence sur le classement. Afin de suivre avec exactitude l'évolution des hospitalisations pour blessure, il est nécessaire d'uniformiser les définitions de cas et les procédures de compilation et de déclaration des données. Notre objectif est de nous pencher davantage sur cet aspect.

\section{Conclusion}

Globalement, les blessures non intentionnelles sont la huitième cause principale d'hospitalisation. Dans le cas des hospitalisations pour blessure non intentionnelle, les autres causes principales sont dans l'ordre les chutes, la suffocation, les accidents de la circulation avec un véhicule à moteur, l'empoisonnement, l'exposition à des forces mécaniques et l'exposition à la fumée, au feu et aux objets brûlants. Toutefois, le classement varie selon le groupe d'âge. L'information actualisée sur les hospitalisations pour blessure est essentielle à la compréhension du fardeau et du profil des blessures au Canada.

\section{Remerciements}

Les auteurs remercient Stephanie Cowle et Pamela Fuselli de Parachute pour leurs précieux commentaires.

\section{Conflits d'intérêts}

Les auteurs déclarent n'avoir aucun conflit d'intérêts.

\section{Contributions des auteurs et avis}

Tous les auteurs ont lu et approuvé le contenu du manuscrit. XY a contribué à la conceptualisation de l'article, à l'analyse des données et à leur interprétation ainsi qu'à la préparation du manuscrit. RS, SM et WT ont contribué à la conceptualisation de l'article, à l'interprétation des données et à la préparation du manuscrit.

Le contenu de l'article et les points de vue qui y sont exprimés n'engagent que les auteurs et ne correspondent pas nécessairement à ceux du gouvernement du Canada.

\section{Références}

1. Haddon W Jr, Suchman EA, Klein D. Accident research: methods and approaches. New York (NY): Harper and Row; 1964.

2. Robertson LS. Injury epidemiology: research and control strategies. 3e édition. New York (NY): Oxford University Press; 2007.

3. Agence de la santé publique du Canada. Étude des blessures, Édition 2012: Pleins feux sur la sécurité routière en matière de transport. Ottawa (Ont.) : Agence de la santé publique du Canada; 2012.

4. Chen Y, Mo F, Yi QL, Jiang Y, Mao Y. La mortalité par blessure non intentionnelle et ses causes externes au Canada entre 2001 et 2007. Maladies chroniques et blessures au Canada. 2013;33(2):110-119.

5. Organisation mondiale de la santé (OMS). Les 10 principales causes de mortalité [Internet]. Genève $(\mathrm{CH})$ : OMS; 2018 [consultation le 21 nov. 2019]. En ligne à : https://www.who .int/fr/news-room/fact-sheets/detail /the-top-10-causes-of-death

6. Organisation mondiale de la santé (OMS). Thèmes de santé : traumatismes [Internet]. Genève $(\mathrm{CH})$ : OMS; 2018 [consultation le 21 nov. 2019]. En ligne à : https://www.who.int /topics/injuries/fr/

7. Statistique Canada. Tableau 13-10-015601, Décès, selon la cause, Chapitre XX : Causes externes de morbidité et de mortalité (V01 à Y89) [Internet]. Ottawa (Ont.) : Statistique Canada; 2019 [consultation le 21 nov. 2019]. En ligne à : https://www150.statcan.gc.ca/t1 /tbl1/fr/tv.action?pid $=1310015601$ 
8. Institut canadien d'information sur la santé. Statistiques sur les visites au service d'urgence et les hospitalisations à la suite d'un traumatisme ou d'une blessure, 2017-2018 [Internet]. Ottawa (Ont.) : Institut canadien d'information sur la santé; 2019 [consultation le 21 nov. 2019]. En ligne à : https://www.cihi.ca/fr/access-data -reports/results?query $=\% 22$ visites $+\mathrm{au}+$ service + d\% E2\% 80\% 99urgence + et + les + hospitalisations + \% C3 $\% \mathrm{~A} 0+\mathrm{la}+$ suite $+\mathrm{d} \% \mathrm{E} 2 \% 80 \% 99 \mathrm{un}$ + traumatisme + ou + d \% E2\% 80\%99 une + blessure $\% 2 \mathrm{C}+2017-2018 \% 22$ $\&$ Search + Submit $=$

9. Parachute. Cost of injury in Canada [Internet]. Toronto (Ont.) : Parachute; 2015 [modification le 31 mai 2019; consultation le 21 nov. 2019]. En ligne à : https://parachute.ca/en/professional -resource/cost-of-injury-report/

10. Parachute. Our leap forward: Parachute's strategic plan, 2018-2020. Toronto (Ont) : Parachute [consultation le 21 nov. 2019]. En ligne à : https:// parachute.ca/en/about-us/our-leap -forward-parachutes-strategic-plan -2018-2020/

11. Yao X, Skinner R, McFaull S, Thompson W. Aperçu - Décès attribuables à des blessures au Canada en 2015. Promotion de la santé et prévention des maladies chroniques au Canada. 2019; 39(6-7):248-254. doi:10.24095/hpcdp $.39 .6 / 7.03 \mathrm{f}$.

12. Agence de la santé publique du Canada. Principales causes d'hospitalisation, Canada, 2009/2010, hommes et femmes confondus, nombre (taux d'hospitalisation selon le groupe d'âge par 100000). Ottawa (Ont.) : Agence de la santé publique du Canada. En ligne à : https://www.canada.ca/fr /sante-publique/services/rapports -publications/principales-causes-deces -hospitalisation-canada/2009-2010 -hommes-femmes-confondus-nombre -taux-hospitalisation-selon-groupe .html

13. Agence de la santé publique du Canada (ASPC). Principales causes d'hospitalisation par blessure, Canada, 2009/2010, nombre (taux d'hospitalisation selon le groupe d'âge par 100 000). Ottawa (Ont.) : ASPC; 2013 [Cat. : HP32 5/2010F-PDF].
14. Institut canadien d'information sur la santé. Codification et classification [Internet]. Ottawa (Ont.) : Institut canadien d'information sur la santé [consultation le 21 nov. 2019]. En ligne à : https://www.cihi.ca/fr/codification -et-classification

15. SAS Institute Inc. SAS Enterprise Guide 7.1. Cary (NC) : SAS Institute Inc.

16. Statistique Canada. Estimations de la population au 1er octobre 2018, par âge, sexe, provinces et territoires. Ottawa (Ont.) : Statistique Canada; 2019.

17. Agence de la santé publique du Canada. Chutes chez les aînés au Canada : Deuxième rapport. Ottawa (Ont.) : Agence de la santé publique du Canada; 2014. En ligne à : https://www .canada.ca/content/dam/phac-aspc /migration/phac-aspc/seniors-aines /publications/public/injury-blessure /seniors_falls-chutes_aines/assets /pdf/seniors_falls-chutes_aines-fra.pdf

18. Parachute. Sujets blessures - Découvrez comment prévenir les blessures avant qu'elles n'arrivent [Internet]. Toronto (Ont.) : Parachute; 2019 [consultation le 21 nov. 2019]. En ligne à : https:// parachute.ca/fr/sujets-blessures/

19. Service canadien de prévention du suicide. Fiche d'information publique de SCC [Internet]. Services de crises du Canada; 2017 [consultation le 21 nov. 2019]. En ligne à : https:// www.crisisservicescanada.ca/fr/faits -rapides/

20. Sécurité publique Canada. Répertoire en prévention du crime. Ottawa (Ont.) : Sécurité publique Canada; 2018 [modification le 21 août; consultation le 21 nov. 2019]. En ligne à : https:// www.securitepublique.gc.ca/cnt/cntrng -crm/crm-prvntn/nvntr/index-fr.aspx $? \mathrm{t}=2 \& \mathrm{SORT}=$ Title\&BY $=$ ASC

21. Ministère de la Sante et des Services sociaux du Québec. MED-ÉCHO - Hospitalisations et chirurgies d'un jour dans les centres hospitaliers du Québec. Québec (QC) : Santé et services sociaux Québec; 2019 [consultation le 21 nov. 2019]. En ligne à : https://www.msss .gouv.qc.ca/professionnels/statistiques -donnees-services-sante-services -sociaux/med-echo-hospitalisations -et-chirurgies-d-un-jour-dans-les -centres-hospitaliers-du-quebec/ 